\title{
Terminkalender 2020/2021
}

https://doi.org/10.1515/iwp-2020-2123

\begin{tabular}{|c|c|c|}
\hline $\begin{array}{l}\text { 5. bis } 7 . \text { Oktober } \\
\text { online }\end{array}$ & $\begin{array}{l}\text { Berliner Herbsttreffen der } \\
\text { Museumsdokumentation }\end{array}$ & $\begin{array}{l}\text { Frank von Hagel, Institut für Museumsforschung, In der Halde 1, } \\
14195 \text { Berlin, Telefon: (030) 8301-460, Fax: (030) 8301-504, } \\
\text { f.v.hagel@smb.spk-berlin.de, www.museumsbund.de }\end{array}$ \\
\hline $\begin{array}{l}\text { 6. bis } 8 \text {. Oktober } \\
\text { online }\end{array}$ & $\begin{array}{l}\text { University: Future Festival - Learning, } \\
\text { Systems and the New Normal } 2020\end{array}$ & $\begin{array}{l}\text { Stifterverband für die Deutsche Wissenschaft e.V., Barkhovenallee 1, } \\
45239 \text { Essen, festival_team@hochschulforum.org }\end{array}$ \\
\hline $\begin{array}{l}\text { 14. Oktober } \\
\text { online }\end{array}$ & $\begin{array}{l}\text { Steilvorlagen für den Unternehmens- } \\
\text { erfolg }\end{array}$ & $\begin{array}{l}\text { GBI-Genios Deutsche Wirtschaftsdatenbank GmbH, Freischützstraße 96, } \\
81927 \text { München, Telefon } 089 \text { 992879-0, info@genios.de }\end{array}$ \\
\hline $\begin{array}{l}\text { 14. bis } 18 . \text { Oktober } \\
\text { Frankfurt am } \\
\text { Main/online }\end{array}$ & $\begin{array}{l}\text { 72. Frankfurter Buchmesse } \\
\text { Ehrengast Kanada }\end{array}$ & $\begin{array}{l}\text { Ausstellungs- und Messe GmbH, Frankfurter Buchmesse, } \\
\text { Braubachstraße 16, 60311 Frankfurt am Main, Telefon } 069 \text { 2102-0, } \\
\text { Fax } 069 \text { 2102-227/-277, info@book-fair.com, www.buchmesse.de }\end{array}$ \\
\hline $\begin{array}{l}\text { 21. Oktober } \\
\text { online }\end{array}$ & $\begin{array}{l}\text { DGI-Praxisseminar: Social Media und } \\
\text { Recherche - Einführung }\end{array}$ & $\begin{array}{l}\text { DGI-Geschäftsstelle, Windmühlstraße 3, } 60329 \text { Frankfurt am Main, } \\
\text { Telefon } 069 \text { 430313, Fax } 069 \text { 4909096, mail@dgi-info.de, } \\
\text { www.dgi-info.de }\end{array}$ \\
\hline $\begin{array}{l}\text { 23. Oktober } \\
\text { Karlsruhe }\end{array}$ & $\begin{array}{l}\text { DTT-Grundlagenseminar: } \\
\text { Terminologiearbeit - Grundlagen, } \\
\text { Werkzeuge, Prozesse }\end{array}$ & $\begin{array}{l}\text { Deutscher Terminologie-Tag e.V. (DTT), Olga Buchstaller-Vodopiyanova, } \\
\text { c/o Technische Hochschule KölnInstitut für Informationsmanagement } \\
\text { (IIM), Gustav-Heinemann-Ufer 54, } 50968 \text { Köln, } \\
\text { geschaeftsstelle@dttev.org }\end{array}$ \\
\hline $\begin{array}{l}\text { 23. bis } 25 . \text { Oktober } \\
\text { online }\end{array}$ & Hackethon Coding Da Vinci & $\begin{array}{l}\text { Wikimedia Deutschland e.V., Tempelhofer Ufer 23-24, } 10963 \text { Berlin, } \\
\text { cdv@tib.eu, www.wikimedia.de, https://codingdavinci.de/events/ } \\
\text { niedersachsen/ }\end{array}$ \\
\hline $\begin{array}{l}\text { 27. Oktober } \\
\text { online }\end{array}$ & $\begin{array}{l}\text { DGI-Praxisseminar: Methoden und } \\
\text { Instrumente der formalen und } \\
\text { inhaltlichen Informationserschließung I }\end{array}$ & $\begin{array}{l}\text { DGI-Geschäftsstelle, Windmühlstraße 3, } 60329 \text { Frankfurt am Main, } \\
\text { Telefon } 069 \text { 430313, Fax } 069 \text { 4909096, mail@dgi-info.de, } \\
\text { www.dgi-info.de }\end{array}$ \\
\hline $\begin{array}{l}\text { 28. Oktober } \\
\text { online }\end{array}$ & $\begin{array}{l}\text { DGI-Praxisseminar: Methoden und } \\
\text { Instrumente der formalen und } \\
\text { inhaltlichen Informationserschließung II }\end{array}$ & $\begin{array}{l}\text { DGI-Geschäftsstelle, Windmühlstraße 3, } 60329 \text { Frankfurt am Main, } \\
\text { Telefon } 069 \text { 430313, Fax } 069 \text { 4909096, mail@dgi-info.de, } \\
\text { www.dgi-info.de }\end{array}$ \\
\hline $\begin{array}{l}\text { 29. bis } 30 . \text { Oktober } \\
\text { online }\end{array}$ & 8. Stuttgarter SharePointForum 2020 & $\begin{array}{l}\text { media science GmbH, Kirchplatz 11, } 70771 \text { Leinfelden-Echterdingen, } \\
\text { kommunikation@stuttgarter-sharepointforum.de }\end{array}$ \\
\hline $\begin{array}{l}\text { 30. Oktober } \\
\text { online }\end{array}$ & $\begin{array}{l}\text { DGI-Praxistage } 2020 \\
\text { Alles, was Recht ist! }\end{array}$ & $\begin{array}{l}\text { DGI-Geschäftsstelle, Windmühlstraße 3, } 60329 \text { Frankfurt am Main, } \\
\text { Telefon } 069 \text { 430313, Fax } 069 \text { 4909096, praxistage2020@dgi-info.de, } \\
\text { www.dgi-info.de }\end{array}$ \\
\hline $\begin{array}{l}\text { 3. November } \\
\text { online }\end{array}$ & $\begin{array}{l}\text { DGI-Praxisseminar: Projektplanung, } \\
\text {-durchführung und -evaluierung I }\end{array}$ & $\begin{array}{l}\text { DGI-Geschäftsstelle, Windmühlstraße 3, } 60329 \text { Frankfurt am Main, } \\
\text { Telefon } 069 \text { 430313, Fax } 069 \text { 4909096, mail@dgi-info.de, } \\
\text { www.dgi-info.de }\end{array}$ \\
\hline $\begin{array}{l}\text { 3. bis } 5 \text {. November } \\
\text { online }\end{array}$ & tekom-Jahrestagung 2020 & $\begin{array}{l}\text { Gesellschaft für technische Kommunikation - tekom Deutschland e.V., } \\
\text { Rotebühlstraße 64, } 70178 \text { Stuttgart, Telefon } 071165704-0, \\
\text { info@tekom.de, www.tekom.de }\end{array}$ \\
\hline $\begin{array}{l}\text { 4. November } \\
\text { online }\end{array}$ & $\begin{array}{l}\text { DGI-Praxisseminar: Projektplanung, } \\
\text {-durchführung und -evaluierung II }\end{array}$ & $\begin{array}{l}\text { DGI-Geschäftsstelle, Windmühlstraße 3, } 60329 \text { Frankfurt am Main, } \\
\text { Telefon } 069 \text { 430313, Fax } 069 \text { 4909096, mail@dgi-info.de, } \\
\text { www.dgi-info.de }\end{array}$ \\
\hline $\begin{array}{l}\text { 4. November } \\
\text { online }\end{array}$ & $\begin{array}{l}\text { h_da-Symposium zur wissenschaftlichen } \\
\text { und Mediendokumentation }\end{array}$ & $\begin{array}{l}\text { Prof. Geribert Jakob, Hochschule Darmstadt, Mediencampus Dieburg, } \\
\text { Max-Planck-Straße 2, } 64807 \text { Dieburg, geribert.jakob@h-da.de }\end{array}$ \\
\hline $\begin{array}{l}\text { 5. November } \\
\text { Essen }\end{array}$ & DIGITAL FUTUREcongress & $\begin{array}{l}\text { AMC Media Network GmbH \& Co. KG, Otto-Hesse-Straße 19, } \\
64293 \text { Darmstadt, Telefon +49 (0) } 6151-957577-0 \text {, } \\
\text { Fax +49 (0) } 6151 \text { - } 957577 \text {-9, office@amc-media-network.de, } \\
\text { www.digital-futurecongress.de }\end{array}$ \\
\hline $\begin{array}{l}\text { 5. bis } 6 . \text { November } \\
\text { Hannover }\end{array}$ & Jahreskonferenz der KIBA & $\begin{array}{l}\text { Prof. Dr. Stefan Schmunk, Hochschule Darmstadt, Mediencampus } \\
\text { Dieburg, Max-Planck-Straße 2, } 64807 \text { Dieburg, stefan.schmunk@h-da.de }\end{array}$ \\
\hline
\end{tabular}




\begin{tabular}{|c|c|c|}
\hline $\begin{array}{l}\text { 10. November } \\
\text { online }\end{array}$ & DGI-Praxisseminar: Wissensmanagement & $\begin{array}{l}\text { DGI-Geschäftsstelle, Windmühlstraße 3, } 60329 \text { Frankfurt am Main, } \\
\text { Telefon } 069 \text { 430313, Fax } 069 \text { 4909096, mail@dgi-info.de, } \\
\text { www.dgi-info.de }\end{array}$ \\
\hline $\begin{array}{l}\text { 9. bis 13. November } \\
\text { online }\end{array}$ & $\begin{array}{l}\text { 16. Stuttgarter Wissensmanagement-Tage } \\
\text { Wissen verbindet }\end{array}$ & $\begin{array}{l}\text { Oliver Lehnert, wissensmanagement - Das Magazin für Digitalisierung, } \\
\text { Vernetzung \& Collaboration, } 86344 \text { Neusäß, Telefon } 0821486852 \text { 92, } \\
\text { Telefax } 0821486 \text { 85293, service@wissensmanagement.net, } \\
\text { www.wissensmanagement.net }\end{array}$ \\
\hline $\begin{array}{l}\text { 12. bis 13. November } \\
\text { Mannheim/online }\end{array}$ & $\begin{array}{l}\text { Wissenschaftliche Bibliotheken gestalten } \\
\text { den digitalen Wandel }\end{array}$ & $\begin{array}{l}\text { Universitätsbibliothek Mannheim, Schloss Schneckenhof West, } \\
68131 \text { Mannheim, Telefon } 0621 \text { 181-2920, } \\
\text { jessica.kaiser@bib.uni-mannheim.de }\end{array}$ \\
\hline $\begin{array}{l}\text { 19. bis 20. November } \\
\text { Hybrid }\end{array}$ & KnowledgeCamp 2020 & $\begin{array}{l}\text { Gesellschaft für Wissensmanagement e. V., https://www.gfwm.de/ } \\
\text { knowledgecamp-2020/, info@gfwm.de }\end{array}$ \\
\hline $\begin{array}{l}\text { 23. bis } 27 . \text { November } \\
\text { online }\end{array}$ & $\begin{array}{l}\text { SWIB19 }-11^{\text {th }} \text { Semantic Web in Libraries } \\
\text { Conference }\end{array}$ & $\begin{array}{l}\text { Joachim Neubert, ZBW-Leibniz-Informationszentrum Wirtschaft, } \\
\text { Düsternbrooker Weg 120, } 24105 \text { Kiel, Telefon } 04042834462 \text {, } \\
\text { j.neubert@zbw.eu, http://swib.org/swib20 }\end{array}$ \\
\hline $\begin{array}{l}\text { 23. November } \\
\text { online }\end{array}$ & $\begin{array}{l}\text { 3. DVMD-Frühjahrssymposium } \\
\text { Datenmanagement, Datenerhebung, } \\
\text { Big Data, Risikobasiertes Monitoring, } \\
\text { Regularien, Biometrie/Statistik }\end{array}$ & $\begin{array}{l}\text { Der Fachverband für Dokumentation und Informationsmanagement } \\
\text { in der Medizin e.v., Lobdengaustraße 1, } 69493 \text { Hirschberg, } \\
\text { dvmd@dvmd.de }\end{array}$ \\
\hline $\begin{array}{l}\text { 27. November } \\
\text { online }\end{array}$ & DGI-Praxisseminar: EU-DSGVO & $\begin{array}{l}\text { DGI-Geschäftsstelle, Windmühlstraße 3, } 60329 \text { Frankfurt am Main, } \\
\text { Telefon } 069 \text { 430313, Fax } 069 \text { 4909096, mail@dgi-info.de, } \\
\text { www.dgi-info.de }\end{array}$ \\
\hline \multicolumn{3}{|l|}{2021} \\
\hline $\begin{array}{l}\text { 11. bis 13. Januar } \\
\text { Berlin }\end{array}$ & APE 2021 Academic Publishing in Europe & $\begin{array}{l}\text { Berlin Institute for Scholarly Publishing (BISP) gGmbH, Genthiner Straße } \\
\text { 13, } 10785 \text { Berlin, www.berlinstitute.org, www.ape2021.eu }\end{array}$ \\
\hline $\begin{array}{l}\text { 2. bis 4. Februar } \\
\text { Karlsruhe }\end{array}$ & $\begin{array}{l}\text { LEARNTEC } 2021 \\
\text { 29. Internationale Fachmesse und } \\
\text { Kongress }\end{array}$ & $\begin{array}{l}\text { Karlsruher Messe- und Kongress-GmbH, Festplatz9, } 76137 \text { Karlsruhe, } \\
\text { Telefon 07213720-0, Fax07213720-2116, info@kmkg.de, } \\
\text { www.learntec.de }\end{array}$ \\
\hline $\begin{array}{l}\text { 17. bis } 18 . \text { Februar } \\
\text { online }\end{array}$ & Open Science Conference 2021 & $\begin{array}{l}\text { Dr. Doreen Siegfried, ZBW-Leibniz-Informationszentrum Wirtschaft, } \\
\text { Düsternbrooker Weg 120, 24105 Kiel, osc@zbw.eu, osc@zbw.eu, } \\
\text { www.open-science-conference.eu/ }\end{array}$ \\
\hline $\begin{array}{l}\text { 25. bis 26. Februar } \\
\text { Leipzig }\end{array}$ & $\begin{array}{l}\text { 16. DVMD-Frühjahrssymposium } \\
\text { Daten verstehen, Forschung unterstützen, } \\
\text { Versorgung verbessern }\end{array}$ & $\begin{array}{l}\text { Der Fachverband für Dokumentation und Informationsmanagement } \\
\text { in der Medizin e.V., Lobdengaustraße 1, } 69493 \text { Hirschberg, } \\
\text { dvmd@dvmd.de }\end{array}$ \\
\hline $\begin{array}{l}\text { 8. bis 10. März } \\
\text { Regensburg }\end{array}$ & $\begin{array}{l}\text { 16. Internationalen Symposium für } \\
\text { Informationswissenschaft } \\
\text { Information zwischen Daten und Wissen: } \\
\text { Die Informationswissenschaft und ihre } \\
\text { Nachbarn von Data Science bis Digital } \\
\text { Humanities }\end{array}$ & $\begin{array}{l}\text { Prof. Dr. Christian Wolff, Universität Regensburg, Institut für Medien-, } \\
\text { Informations- und Kulturwissenschaft (IMIK), Universitätsstraße 31, } \\
93053 \text { Regensburg, christian.wolff@sprachlit.uni-regensburg.de, } \\
\text { www.isi2021.de }\end{array}$ \\
\hline $\begin{array}{l}\text { 6. bis } 9 . \text { September } \\
\text { Amsterdam, } \\
\text { Niederlande }\end{array}$ & $\begin{array}{l}\text { SEMANTICS } 2021 \text { - In the Era of } \\
\text { Knowledge Graphs }\end{array}$ & $\begin{array}{l}\text { Semantic Web Company, R\&I Department, Neubaugasse 1, } 1070 \text { Wien, } \\
\text { Österreich, https://2019.semantics.cc/conference }\end{array}$ \\
\hline $\begin{array}{l}\text { 20. bis 24. Oktober } \\
\text { Frankfurt am Main }\end{array}$ & $\begin{array}{l}\text { 73. Frankfurter Buchmesse } \\
\text { Ehrengast Spanien }\end{array}$ & $\begin{array}{l}\text { Ausstellungs- und Messe GmbH, Frankfurter Buchmesse, } \\
\text { Braubachstraße 16, 60311 Frankfurt am Main, Telefon } 069 \text { 2102-0, } \\
\text { Fax } 069 \text { 2102-227/-277, info@book-fair.com, www.buchmesse.de }\end{array}$ \\
\hline $\begin{array}{l}\text { 24. bis } 25 . \text { Oktober } \\
\text { Berlin }\end{array}$ & $\begin{array}{l}\text { Kongress des International Committee of } \\
\text { Representatives of Indexing Societies } \\
\text { (ICRIS) }\end{array}$ & $\begin{array}{l}\text { DGI-Geschäftsstelle, Windmühlstraße 3, } 60329 \text { Frankfurt am Main, } \\
\text { Telefon } 069 \text { 430313, Fax } 069 \text { 4909096, mail@dgi-info.de, } \\
\text { www.dgi-info.de }\end{array}$ \\
\hline $\begin{array}{l}\text { 26. bis 28. Oktober } \\
\text { Bern, Schweiz }\end{array}$ & Schweizer Bibliothekskongress 2021 & $\begin{array}{l}\text { Bibliosuisse, Bleichemattstrasse 42, } 5000 \text { Aarau, Schweiz, } \\
\text { Telefon +41 } 62823 \text { 1938, Fax +41 } 62823 \text { 1939, info@bibliosuisse.ch, } \\
\text { info@bis.ch }\end{array}$ \\
\hline
\end{tabular}

